50 | avril 2009

En classe : pratiques pédagogiques et valeurs culturelles

\title{
Une sélection de ressources en ligne sur l'éducation en Afrique subsaharienne
}

\section{Sophie Condat}

\section{(2) OpenEdition \\ Journals}

Édition électronique

URL : http://journals.openedition.org/ries/472

DOI : $10.4000 /$ ries.472

ISSN : 2261-4265

Éditeur

Centre international d'études pédagogiques

Édition imprimée

Date de publication : 1 avril 2009

Pagination : 11-14

ISSN : 1254-4590

\section{Référence électronique}

Sophie Condat, "Une sélection de ressources en ligne sur l'éducation en Afrique subsaharienne », Revue internationale d'éducation de Sèvres [En ligne], 50 | avril 2009, mis en ligne le 01 juillet 2011, consulté le 06 janvier 2020. URL : http://journals.openedition.org/ries/472 ; DOI : 10.4000/ries.472

Ce document a été généré automatiquement le 6 janvier 2020

(c) Tous droits réservés 


\title{
Une sélection de ressources en ligne sur l'éducation en Afrique subsaharienne
}

\author{
Sophie Condat
}

1 L'article s'inscrit dans la continuité des ressources en ligne consacrées à l'éducation par zone géographique, après l'Amérique latine et les Caraïbes ( $\mathrm{n}^{\circ} 45$, septembre 2007) et l'Asie ( ${ }^{\circ} 48$, septembre 2008). Nous avons sélectionné des organismes internationaux et régionaux intervenant dans le domaine éducatif en Afrique, des institutions financières, un organisme de recherche et des sites sur l'intégration des TIC en Afrique.

2 Sitographie arrêtée le 23 février 2009.

\section{Organismes}

\section{Organismes internationaux}

Institut international de planification de l'éducation (IIPE)

Centre de formation et de recherche de l'Unesco spécialisé en planification et gestion de l'éducation, l'IIPE a pour objectif d'aider les pays à développer leurs systèmes éducatifs. Son site (en français, anglais et espagnol) donne accès à la liste des projets menés en Afrique en 2006-2007, où l'institut est particulièrement actif, et à ses publications. Planipolis, portail des plans et politiques d'éducation des États membres de l'IIPE, met en ligne des documents (politiques nationales d'éducation, plans nationaux d'éducation dont ceux pour l'éducation pour tous (EPT), rapports sur les objectifs pour le millénaire du développement OMD) émanant de sources officielles. La recherche s'effectue par zone géographique, mot clé et type de document. www.iiep.unesco.org/fr.html 


\section{Bureau international d'éducation (BIE)}

4 Centre de l'Unesco spécialisé dans le développement des contenus éducatifs, le BIE met en ligne (en anglais, français, espagnol, russe, arabe et chinois) des informations sur les systèmes éducatifs et les curricula, les programmes, projets et activités en cours; des ressources (rapports nationaux, dossiers par pays); ses bases de données; ses publications. La rubrique « Dossiers pays » (entrée par région ou par pays) propose des données sur les systèmes d'enseignement, les curricula et une sélection de liens vers des sources officielles d'information sur l'éducation.www.ibe.unesco.org/fr/dans-lemonde/afrique.html

\section{Organismes régionaux}

\section{Bureau régional de l'UNESCO pour l'Afrique (BREDA)}

5 Représentant l'UNESCO auprès de six pays (Cap Vert, Gambie, Guinée-Bissau, Liberia, Sénégal, Sierra Leone), le BREDA est chargé de planifier l'éducation en Afrique subsaharienne et de coordonner la mise en œuvre des programmes éducatifs régionaux. Son intervention couvre l'éducation de base, l'enseignement secondaire et technique, l'enseignement supérieur, les politiques éducatives et l'éducation préventive: des documents sur ces thématiques sont en ligne (en français, anglais, portugais). www.dakar.unesco.org/

\section{Pôle de Dakar}

6 Le Pôle de Dakar (BREDA) développe une expertise en analyse sectorielle de l'éducation au service des pays africains et de leurs partenaires, de l'élaboration à la mise en œuvre des politiques éducatives. Ses publications - rapports de synthèse régionaux, rapports d'état d'un système éducatif national (RESEN), notes pays et thématiques - sont en ligne (français, anglais). Ainsi que ses contributions à l'EPT, à l'initiative Fast Track (mise en œuvre accélérée de l'EPT) et à l'amélioration de la gestion de l'éducation en Afrique. Une entrée par pays donne accès à plusieurs types d'indicateurs (financiers, EPT, cycle primaire). www.poledakar.org/

\section{Association pour le développement de l'éducation en Afrique (ADEA)}

Plateforme d'échange sur les politiques éducatives en Afrique mais aussi réseau de décideurs, de praticiens et de chercheurs, l'ADEA se veut un appui aux pays engagés dans la réforme de leurs systèmes éducatifs. L'un de ses objectifs est de renforcer les liens entre les ministères africains de l'éducation et de la formation et les organismes de coopération et de développement. Elle vise aussi à développer les capacités institutionnelles et techniques africaines en établissant des réseaux d'échange d'informations. Ses programmes et activités (biennales, publications, bases de données, études de cas) sont en ligne (français, anglais). www.adeanet.org/

\section{Association des universités africaines (AAU)}

8 Forum de consultation, d'échange et de coopération entre les institutions d'enseignement supérieur en Afrique, l'AUU (45 pays) a pour mission d'améliorer la 
qualité de l'enseignement supérieur en soutenant notamment la formation de réseaux d'enseignement, de recherche et de diffusion d'informations par les institutions de l'enseignement supérieur. Elle représente la voix de l'enseignement supérieur en Afrique au niveau des organes régionaux et internationaux. Ses publications et rapports annuels sont en ligne (français, anglais). www.aau.org/

\section{Programmes}

\section{Le Nouveau partenariat pour le développement de l'Afrique (NEPAD)}

Programme de l'Union africaine adopté par les chefs d'États et de gouvernements africains, le NEPAD veut contribuer à la renaissance de l'Afrique en luttant contre la pauvreté et le sous-développement. En éducation, l'un de ses secteurs d'activités prioritaire, il a pour but de collaborer avec les bailleurs de fonds et les institutions multilatérales pour réaliser l'objectif d'éducation primaire universelle d'ici l'an 2015; d'améliorer la qualité de l'enseignement, de l'élaboration et de la réforme des programmes; d'élargir l'accès à l'enseignement secondaire et aux nouvelles technologies; de favoriser la mise en place de réseaux de recherche pour l'enseignement supérieur. Ses plans d'action et projets sont en ligne (en français et en anglais). www.nepad.org/

\section{Conférence des ministres de l'éducation ayant le français en partage (Confemen)}

10 Institution de la francophonie, la Confemen (41 États et gouvernements membres, dont de nombreux africains) œuvre pour la promotion de l'éducation et de la formation professionnelle et technique. Le Programme d'analyse des systèmes éducatifs des États et gouvernements membres (PASEC) a pour objet d'évaluer les systèmes éducatifs pour améliorer l'accès à une éducation de qualité pour tous. Les rapports et documents du PASEC sont en ligne. www.confemen.org

\section{Institutions financières}

\section{Banque mondiale}

11 Organisme international, la Banque mondiale apporte un appui financier et technique aux pays en développement. Elle soutient de nombreux projets, notamment en éducation. Pour la troisième année consécutive, l'Afrique est sa région d'intervention prioritaire. Son site (en français, anglais et espagnol) présente les projets et programmes de développement qu'elle soutient, ses publications (rapports régionaux ou nationaux) ainsi que des données statistiques. La rubrique «Profils pays » donne accès à des données éducatives par pays (synthèses, indicateurs internationaux, statistiques) et permet de connaître les activités de la Banque mondiale dans une région ou un pays (projets/programmes, publications, rapports). www.banquemondiale.org/ 


\section{Groupe de la Banque africaine de développement (BAD)}

12 La BAD est une banque de développement régionale et multilatérale dont le principal objectif est de promouvoir une croissance économique durable et de réduire la pauvreté en Afrique. Elle finance un large éventail de projets et programmes nationaux et multinationaux, dont certains axes concernent le secteur éducatif. L'entrée " Pays » (français, anglais) permet d'accéder aux documents clés d'un pays (documents de stratégies pays, NEPAD, etc.) ainsi qu'à une sélection de liens. La rubrique «Documents» donne accès, par pays et par thématique, à d'autres informations (rapports, projets). www.afdb.org/

\section{L'Agence Française de Développement (AFD)}

13 Au cœur du dispositif français d'aide publique en faveur des pays pauvres, l'AFD participe au financement du développement. En éducation, l'un de ses principaux secteurs d'intervention, elle finance l'ensemble des programmes bilatéraux français pour l'éducation primaire, secondaire et la formation professionnelle. Plus de $50 \%$ de ses financements sont destinés à l'Afrique. Les projets qu'elle soutient sont en ligne (français, anglais, espagnol) : projets en cours, quelques projets emblématiques, projets d'une zone géographique ou d'un pays. Un lien donne accès à ses études et publications. www.afd.fr/

\section{Organisme de recherche}

\section{Réseau Ouest et Centre Africain de Recherche en Éducation (ROCARE)}

Il vise à promouvoir l'expertise africaine pour améliorer les politiques et pratiques éducatives via, par exemple, le renforcement de la capacité nationale et régionale de recherche et d'évaluation. Le réseau compte plusieurs centaines de chercheurs dans quatorze pays membres (Bénin, Burkina Faso, Cameroun, Côte d'Ivoire, Gambie, Ghana, Guinée, Mali, Mauritanie, Niger, Nigéria, Sénégal, Sierra Leone, Togo). Ses publications sont en ligne (bulletins d'information, newsletters, études nationales et transnationales, etc.). www.rocare.org/

\section{TICE : ressources, enseignement à distance}

\section{Institut international pour le renforcement des capacités en Afrique (IIRCA)}

Institut de l'UNESCO, l'IIRCA vise à renforcer le poids des structures d'enseignement en Afrique, notamment par le biais des nouvelles technologies avec la mise en place d'une plate-forme d'échange et de ressources pédagogiques pour les enseignants. www.unesco-iicba.org/ 


\section{Edusud - TIC pour l'éducation en Afrique}

Élaboré par le pôle TIC et Éducation du BREDA, le portail Edusud se veut un espace de savoirs pour l'intégration des TIC en Afrique. Il propose aux enseignants des outils et ressources pédagogiques (méthodes, logiciels, annuaires de recherche) dans le domaine des TIC et donne accès aux sites des universités francophones proposant des cours en ligne. www.edusud.org/

\section{INDEX}

Mots-clés : politique éducative, TICE : technologies de l'information et de la communication en éducation

Index géographique : Afrique subsaharienne

Keywords : educational policy, information and communication technologies in education Palabras claves : política educacional, tecnologías de la información y la comunicación en la educación

\section{AUTEUR}

\section{SOPHIE CONDAT}

Documentaliste au centre de ressources documentaires du CIEP. 